\title{
Response of human cancer cells to simultaneous treatment with sorafenib and radiofrequency current
}

\author{
MARÍA LUISA HERNÁNDEZ-BULE, MARÍA ANTONIA MARTÍNEZ, MARÍA ÁNGELES TRILLO, \\ LIDIA MARTÍNEZ, ELENA TOLEDANO-MACÍAS and ALEJANDRO ÚBEDA \\ Bioelectromagnetism-Research Service, Ramón y Cajal University Hospital, IRYCIS, Madrid 28034, Spain
}

Received February 16, 2021; Accepted August 24, 2021

DOI: 10.3892/ol.2021.13068

\begin{abstract}
Due to their alleged analgesic, anti-inflammatory and tissue regenerative effects, capacitive-resistive electrothermal therapy (CRET), which is based on non-invasive exposure to radiofrequency (RF) currents, is often applied to chemotherapeutically treated patients with cancer. Our previous studies have demonstrated that subthermal CRET currents can elicit a number of cell responses, including anti-proliferative effects, in the human liver cancer cell line HepG2. Such effects involve significant changes in the regulation of proteins involved in MAPK signaling pathways, which are also implicated in the cancer cell response to standard anticancer drugs such as sorafenib. This overlap in response pathways may lead to competitive, neutralizing or blocking interactions between the electrical and chemical treatments, thus raising questions on the advisability of CRET treatment for their analgesic, anti-inflammatory or other purposes in patients undergoing chemotherapy. The present study analyzed the effects of simultaneous treatment with sorafenib and $448-\mathrm{kHz}$, subthermal CRET current on the proliferation and viability of HepG2 cell cultures. Cell viability was assessed through Trypan blue or XTT assays, while flow cytometry was applied for cell cycle and apoptosis analysis. The expression of proteins involved in cell proliferation were assessed by immunoblotting and immunofluorescence. The results revealed no evidence to suggest that the electrical treatment counteracted or neutralized the cellular response to sorafenib at the different conditions evaluated. Furthermore, at the standard pharmacological sorafenib concentration, $5 \mu \mathrm{M}$, the combined treatment elicited an anti-proliferative response significantly stronger than that induced by each of the treatments when applied separately in HepG2 cells. These data do not support the hypothesis that CRET exposure may inhibit or diminish the effects of a chemotherapeutic drug used in
\end{abstract}

Correspondence to: Dr María Luisa Hernández-Bule, Bioelectromagnetism-Research Service, Ramón y Cajal University Hospital, IRYCIS, 9-100 km Colmenar Viejo Road, Madrid 28034, Spain E-mail: mluisa.hernandez@hrc.es

Key words: electrotherapy, HepG2 cells, sorafenib, cell cycle, EGFR cancer treatment, and highlights the requirement for further investigation into the cell response to the combined action of electrical and chemical treatments.

\section{Introduction}

Capacitive-resistive electrothermal therapy (CRET) is based on the non-invasive application of $0.45-0.60 \mathrm{MHz}$ radiofrequency $(\mathrm{RF})$ currents that, when circulating through the treated tissues, causes a temperature increase due to ion reorientation and friction (1). This therapy has been applied successfully for skin, muscle and osteoarticular tissue repair (2,3), as well as for the treatment of arthritis (4), Peyronie's disease (5) and ailments involving poor blood flow $(6,7)$.

There are indications that the induction of cellular responses of a subthermal or microthermal nature could also play a role in the therapeutic effects of these RF currents. Indeed, our previous studies have revealed that electrical currents used in CRET therapies, when applied in vitro at subthermal densities, could induce significant effects on the proliferation (8), differentiation $(9,10)$ or viability of different human cell types (1). These effects, which differ between distinct cell types and are non-linearly dependent on RF signaling parameters, such as frequency, modulation or current density, were found to be mediated by electrically-induced changes in the regulation of proteins involved in the aforementioned processes $(11,12)$. In cancer cells, CRET currents have been shown to induce anti-proliferative and/or cytotoxic responses (11,13-15).

The Ras/Raf/RAF/MEK/ERK signaling pathway plays an essential role in the regulation of liver cell proliferation (16), and alterations in this pathway have been reported to be involved in the promotion of hepatocellular carcinoma (HCC) (17). Thus, inhibition of the Ras/ERK signaling pathway is considered a plausible approach for HCC treatment. In this regard, the multikinase inhibitor, sorafenib, is the only systemically applicable chemotherapeutic drug approved by the United States Food and Drug Administration for the standardized treatment of HCC $(18,19)$. Sorafenib acts predominantly through the inhibition of pathways involved in cell survival and angiogenesis, such as the VEGFR or the platelet-derived growth factor receptor (PDGFR) pathways (20), as well as the Raf kinases pathway, in which sorafenib inhibits MEK and ERK phosphorylation (16). In the human cell line HepG2, CRET exerts anti-proliferative and differentiating effects through 
changes in the regulation of various proteins, including cyclins, cyclin-dependent kinase inhibitors or kinases of MAPK pathways such as ERK1/2 (1,9,21).

Thus, both CRET and sorafenib have been found to inhibit HepG2 cell proliferation through their effects on members of the Ras/ERK signaling pathway. This crosstalk between the electrical and chemical response pathways leads to the possibility of competitive, neutralizing or blocking interactions between both types of treatment. The potential existence of interactions of this nature raises doubts about the advisability of patients with cancer being treated with CRET for analgesic, anti-inflammatory, cicatrization or other purposes, while undergoing chemotherapy. The present study aimed to analyze the in vitro effects of simultaneous treatment with sorafenib and 448-kHz CRET electrical current on the proliferation and viability of the cell line HepG2. The obtained results revealed no evidence to suggest that the electrical treatment counteracted or neutralized the cellular response to sorafenib in the different conditions tested. On the contrary, under certain conditions, the combined treatment produced a significantly stronger anti-proliferative response than that induced by each of the treatments when applied individually.

\section{Materials and methods}

Cell culture. The human liver cancer cell line, HepG2, was obtained from the European Collection of Authenticated Cell Cultures (cat. no. 85011430). The cells were seeded into $75-\mathrm{cm}^{2}$ T-flasks (Falcon; Corning Life Sciences) in DMEM (Gibco; Thermo Fisher Scientific, Inc.) supplemented with $10 \%$ (vol/vol) fetal bovine serum (FBS; Gibco; Thermo Fisher Scientific, Inc.), 4 mM L-glutamine (Gibco; Thermo Fisher Scientific, Inc.) and $100 \mathrm{U} / \mathrm{ml}$ penicillin-streptomycin with fungizone (Gibco; Thermo Fisher Scientific, Inc.), and incubated in a $5 \% \mathrm{CO}_{2}$ atmosphere at $37^{\circ} \mathrm{C}$. The cultures were trypsinized and sub-cultured once a week.

Treatment procedures. The procedure and materials for CRET exposure have been described in previous studies $(1,13)$. Briefly, the cells were seeded in 60-mm Petri dishes (Nunc; Thermo Fisher Scientific, Inc.) at a density of $10^{5}$ cells $/ \mathrm{ml}$. The plates were divided into four groups: Sham-treated control cells incubated in the presence of unenergized electrodes, cells treated with CRET only, cells treated with sorafenib only and cells simultaneous treated with sorafenib + CRET. On day 4 after seeding, when $70-80 \%$ confluence was reached, the cultures were subjected to electrical or chemical treatment or both. Based on previously reported data on the response of HepG2 cells to sorafenib $(22,23)$, cells were treated with sorafenib for 48 hours at concentrations of $3 \mu \mathrm{M}, 5 \mu \mathrm{M}$ or $7 \mu \mathrm{M}$, using DMSO (Gibco; Thermo Fisher Scientific, Inc.) at a 1:1,000 dilution in DMEM with 10\% FBS as a vehicle.

For simultaneous electrical and chemical treatment, on day 4 post-seeding, after adding sorafenib, stainless steel electrodes designed ad hoc for CRET treatment were inserted in all plates, both treated and controls. Treatment with sinewave current at $448 \mathrm{kHz}$ and $50 \mu \mathrm{A} / \mathrm{mm}^{2}$ was applied cyclically ( $5 \mathrm{~min}$ on/235 min off) for a total of $24 \mathrm{~h}$, using a CRET system Indiba Activ HCR 902 power supply (Indiba S.A.). All cultures were then incubated for an additional $24 \mathrm{~h}$ in the absence of electrical stimulation. For the western blotting experiments, the samples received a short, $4-\mathrm{h}$ exposure to the combined treatment. At the end of the chemical and/or electrical treatments, the samples were processed for the corresponding assays.

Analysis of cell viability by quantification with Trypan Blue. The cells were trypsinized with a $0.25 \%$ solution of trypsine (Gibco; Thermo Fisher Scientific, Inc.) and diluted in $1 \mathrm{ml}$ of supplemented DMEM culture medium. Aliquots of this solution were stained with 0.4\% Trypan Blue (Sigma-Aldrich; Merck KGaA) diluted 1:4 in PBS (Gibco) and counted in a Neubauer chamber for assessment of cell viability.

XTT viability assay. Cell viability was determined by XTT assay (Roche Diagnostics GmbH). After sham, electrical, chemical or simultaneous treatments, the cells were incubated for $3 \mathrm{~h}$ with the tetrazolium salt XTT in a $37^{\circ} \mathrm{C}$ and $6 \% \mathrm{CO}_{2}$ atmosphere, as recommended by the manufacturer. The metabolically active cells reduced XTT into coloured formazan compounds that were quantified with a microplate reader (Tecan) at a $492 \mathrm{~nm}$ wavelength.

Immunofluorescence evaluation of the proliferation marker Ki67. An immunofluorescence assay for Ki67 was carried out on cells cultured on coverslips. At the end of the 48-h treatment period, the cells were fixed with $4 \%$ paraformaldehyde and permeabilized with $95 / 5$ ( $\mathrm{vol} / \mathrm{vol}$ ) ethanol/acetic acid. The cells were incubated overnight at $4{ }^{\circ} \mathrm{C}$ with the monoclonal primary antibody, anti-Ki67 (SP6; 1:250, cat. no. ab16667; Abcam). Next, the secondary antibody, Alexa Fluor ${ }^{\circledR}$ 488-conjugated goat anti-rabbit IgG (1:500; cat. no. A11034; Thermo Fisher Scientific, Inc.) was added, and the samples were incubated at room temperature for $1 \mathrm{~h}$. The preparations were counterstained, mounted in ProLong ${ }^{\mathrm{TM}}$ Gold antifade reagent with DAPI (cat. no. P36941; Thermo Fisher Scientific, Inc.), and observed through an inverted fluorescence microscope (Nikon Eclipse Ts2R; Nikon Corporation) attached to a digital camera DS-Ri2 (Nikon Corporation). Images from three experimental replicates were recorded, and $\mathrm{Ki}_{67^{+}}$cells as well as total cells were counted with NIS-Elements Br image software (version 4.40; Nikon Corporation). $\mathrm{Ki}^{+}{ }^{+}$cell identification was based on fixed thresholds of fluorescence determined and automated at the beginning of the analysis. In each experimental repeat, 15 microscope fields were analyzed per experimental condition.

Cell cycle analysis by flow cytometry using propidium iodide. After trypsinization, the cells were centrifuged, resuspended in $70 \%$ ethanol and fixed at $4^{\circ} \mathrm{C}$ for $\geq 24 \mathrm{~h}$. To detect apoptotic cells that could be suspended in the culture medium, the media from all plates were collected and centrifuged. The resulting pellets were processed together with the rest of the cells on the plates. For propidium iodide labeling, the cells were resuspended and stained with a solution of $3.4 \mathrm{mM}$ sodium citrate (PanReac Quimica SLU), $100 \mu \mathrm{g} / \mathrm{ml}$ RNAse A (Roche Diagnostics $\mathrm{GmbH}$ ) and $20 \mu \mathrm{g} / \mathrm{ml}$ propidium iodide (Roche Diagnostics $\mathrm{GmbH}$ ), and incubated in the dark at room temperature for $1 \mathrm{~h}$.

For data acquisition, a total of 20,000 events were counted using FACScan Mod and FACScalibur flow cytometer 
(BD Biosciences). The obtained data were analyzed using CellQuest 3.2 software (BD Immunocytometry Systems).

Assessment of proliferating cell nuclear antigen (PCNA), cyclin D1, phosphorylated p-ERK1/2, ERK1/2, p-EGFR and EGFR expression by western blotting. At the end of each experimental replicate, the cell samples were centrifuged and lysed in lysis buffer containing $10 \mathrm{mM}$ Tris $\mathrm{HCl}$ (Merck KGaA) pH 7.6, $10 \mathrm{mM} \mathrm{KCl}$ (Merk KGaA), 1 mM dithiothreitol (Sigma-Aldrich; Merck KGaA), 1 mM EDTA (Bio-Rad Laboratories, Inc.), $1 \mathrm{mM}$ PMSF (Sigma-Aldrich; Merck KGaA), $10 \mu \mathrm{g} / \mathrm{ml}$ leupeptin (Sigma-Aldrich; Merck KGaA), $5 \mu \mathrm{g} / \mathrm{ml}$ pepstatin (Sigma-Aldrich; Merck KGaA), 100 mM NaF (Sigma-Aldrich; Merck KGaA), $20 \mathrm{mM} \beta$-glycerophosphate (Calbiochem; Merck KGaA), $20 \mathrm{mM}$ sodium molybdate (Sigma-Aldrich; Merck KGaA), 0.5\% Triton X-100 (ICN Biomedicals, Inc.) and 0.1\% SDS (Bio-Rad Laboratories, Inc.). Protein concentration was determined using a Pierce BCA Protein assay (Thermo Fisher Scientific, Inc.). Next, protein samples (100- $\mu$ g protein aliquots) were separated in 8-10\% SDS-PAGE and electrophoretically transferred onto nitrocellulose membranes (Amersham; Cytiva) using a semi-dry system (Trans-Blot ${ }^{\circledR}$ SD semi-dry transfer cell; Bio-Rad Laboratories, Inc.).

The blots were incubated at $4^{\circ} \mathrm{C}$ overnight in the presence of the following antibodies: Mouse monoclonal primary anti-cyclin D1 (1:1,000; cat. no. P2D11F11; Novocastra Laboratories Ltd.), mouse monoclonal anti-PCNA (1:1,000; cat. no. sc-56; Santa Cruz Biotechnology, Inc.), rabbit polyclonal primary antibody anti-p-EGFR (1:1,000; cat. no. 3777; Cell Signaling Technology, Inc.), mouse monoclonal primary antibody anti-EGFR (1:1,000; cat. no. MA5-13877; Thermo Fisher Scientific, Inc.), rabbit polyclonal primary anti-ERK1/2 (1:1,000; cat. no. 9102S; Thermo Fisher Scientific, Inc.) and rabbit polyclonal primary antibody p-ERK1/2 (1:1,000; cat. no. 44-680G; Thermo Fisher Scientific, Inc.). Monoclonal mouse anti- $\beta$-actin antibody (1:5,000; A5441; Sigma-Aldrich; Merck KGaA) was used as the loading control. To detect non-phosphorylated forms of ERK1/2, the membranes were stripped with $25 \mathrm{mM}$ glycine at $\mathrm{pH} 2.0$ for $30 \mathrm{~min}$. The proteins of interest were detected using horseradish peroxidase-conjugated secondary antibodies during $1 \mathrm{~h}$ at room temperature (ECL donkey anti-rabbit; 1:3,000; cat. no. NA934; sheep anti-mouse; 1:5,000; cat. no. NA931; or IgG horseradish peroxidase-linked species-specific whole antibody; all from Cytiva) or a fluorescently labeled secondary antibody (IRDye ${ }^{\circledR} 800$ CW goat anti-rabbit polyclonal secondary antibody; 1:10,000; cat. no. 926-32211; LI-COR Biosciences) in same incubation conditions. ChemiDoc Imaging system (Bio-Rad Laboratories, Inc.) was used to detect ECL in the blots, while detection of phosphorylated forms of EGFR (p-EGFR) was carried out through immunofluorescence development with Odyssey system (LI-COR Biosciences). All images were analyzed with Quantity One software (version 4.6.7; Bio-Rad Laboratories, Inc.).

Statistical analysis. Statistical differences between groups were determined by one-way ANOVA followed by Bonferroni post-hoc tests or via an unpaired Student's t-test, using GraphPad Prism 6.01 software (GraphPad Software, Inc.). At least three independent replicates were conducted per experiment and exposure interval. Results were expressed as the mean \pm SD or SEM. $\mathrm{P}<0.05$ was considered to indicate a statistically significant difference.

\section{Results}

Effects of sorafenib on cell viability when administered at different concentrations. The preselection of sorafenib concentrations suitable for the combined treatment with CRET was carried out using the Trypan Blue exclusion assay for cell viability. After $48 \mathrm{~h}$ of treatment, sorafenib inhibited cell viability and increased cell death in a dose-dependent manner (Fig. 1A and B). The half-maximal inhibitory concentration $\left(\mathrm{IC}_{50}\right)$ of sorafenib, as determined by non-lineal regression analysis using Excel software (Microsoft Excel 2013, 15.0.4945.1000, 32 bits), was $7 \mu \mathrm{M}$ (Fig. 1C).

Effects of CRET and sorafenib on cell viability. The effects of the chemical and electrical treatments, administered individually or in combination, were quantified with Trypan Blue $48 \mathrm{~h}$ after initiation of the treatment. The results of the Trypan Blue assay (Fig. 1D) showed that, when applied individually, both CRET exposure and chemical treatment at different concentrations significantly reduced cell viability compared with that of the corresponding controls. In the combined treatment, CRET did not significantly alter the reduction in cell viability induced by 3 or $7 \mu \mathrm{M}$ sorafenib. However, at the intermediate concentration of $5 \mu \mathrm{M}$, which, when applied alone, induced a decrease in viability equivalent to that elicited by CRET alone ( $\sim 85 \%$ of the value in the controls), combined exposure resulted in a decrease in viability significantly greater than that induced by chemical treatment $(\sim 70 \%$ of the value in the controls).

XTT results showed in supplementary data (Fig. S1) revealed that this technique is less sensitive than Trypan Blue for detecting the effects of CRET on the viability of HepG2 cells. This poor sensitivity of the XTT assay has been previously reported when describing the effects of anticancer drugs such as sorafenib (which is capable of inducing the generation of reactive oxygen species) on the viability of HepG2 cells (24). Based on these results, the use of XTT and other assays that evaluate cellular metabolic function through reduction of tetrazolium salts to formazan was discarded in the present study. The subsequent experiments were focused on the response of HepG2 cells to CRET exposure in the presence of $5 \mu \mathrm{M}$ sorafenib $\left(\mathrm{IC}_{15}\right)$.

Effects of CRET and sorafenib on the expression of proteins involved in cell proliferation. The effects of CRET and sorafenib on cell proliferation were assessed by PCNA and Ki67 protein expression analysis. PCNA expression was analyzed at 4 and $48 \mathrm{~h}$ of treatment. At $4 \mathrm{~h}$, only the samples subjected to the combined treatment showed a non-significant decrease in PCNA expression compared with that of the controls. At $48 \mathrm{~h}$, the samples treated with sorafenib, either in the presence or absence of CRET stimulation, showed equivalent, statistically significant reductions in PCNA expression. When applied alone, the electrical treatment did not significantly affect the expression of PCNA after 4 or $48 \mathrm{~h}$ of exposure (Fig. 2A and B). After 2 days of treatment, the 

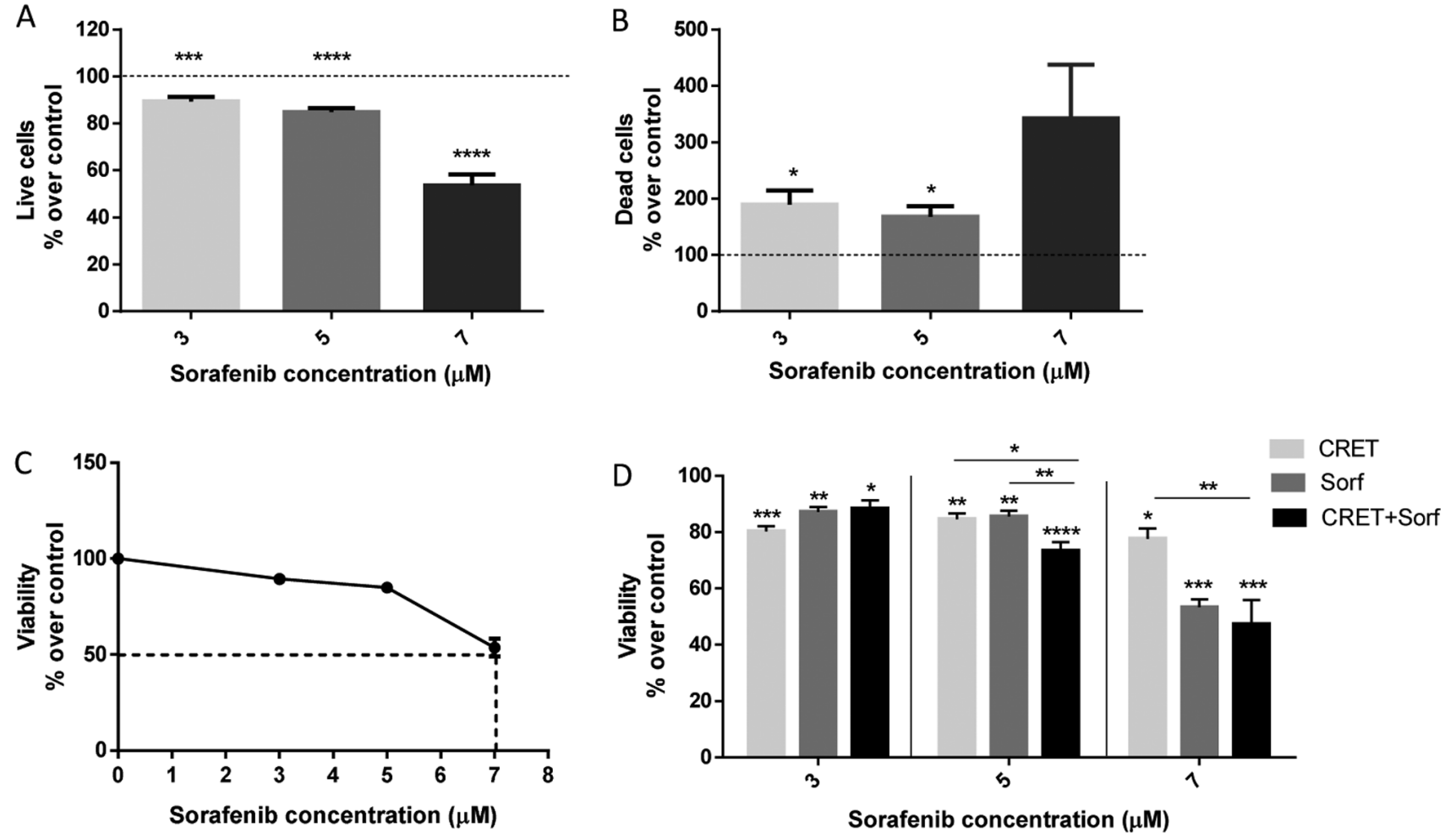

Figure 1. Effects of treatment with CRET and/or sorafenib on HepG2 cell viability. (A) Live and (B) dead cells after 48 h of treatment with different concentrations of sorafenib. (C) Half-maximal inhibitory concentration determination. (D) Cell viability after treatment with CRET alone $\left(50 \mu \mathrm{A} \mathrm{mm}{ }^{2} ; 24 \mathrm{~h}\right.$ of intermittent exposure $+24 \mathrm{~h}$ post-exposure), sorafenib alone $(3-7 \mu \mathrm{M} ; 48 \mathrm{~h})$ or combined (CRET + sorafenib). Data are presented as the mean \pm SD of $\geq 3$ experimental replicates per concentration. The values were normalized to the corresponding controls. ${ }^{*} \mathrm{P}<0.05,{ }^{* * *} \mathrm{P}<0.01,{ }^{* * * *} \mathrm{P}<0.001$ and ${ }^{* * * * *} \mathrm{P}<0.0001$. Data were statistically analyzed via One-way ANOVA followed by Bonferroni post-hoc test. CRET, capacitive-resistive electrothermal therapies; Sorf, sorafenib.

number of cells expressing the proliferation marker, Ki67, was significantly decreased in the samples subjected to the combined treatment and in those exposed separately to the chemical or physical treatment compared with that in the controls. The effect of combined treatment was significantly stronger than that of CRET only (Fig. 2C and D).

Effects of CRET and sorafenib on apoptosis and cell cycle. In order to identify processes underlying the observed decrease in cell viability, the potential effects of the aforementioned treatments on cell cycle or apoptosis were analyzed by flow cytometry using propidium iodide staining. The data summarized in Fig. 3 revealed that the apoptosis rate (phase sub- $\mathrm{G}_{0} / \mathrm{G}_{1}$ ) was not significantly increased in response to separate treatments with CRET or $5 \mu \mathrm{M}$ sorafenib, as well as to combined treatment. However, the biological relevance of these potential proapoptotic effects is expected to be rather limited, since the typical rates of spontaneous apoptosis in the HepG2 cell line are markedly low (1.3\% in the controls). Cell cycle analysis revealed that the electrical treatment reduced, although not significantly, the proportion of cells in the $S$ phase. Sorafenib when administered alone or in combination with CRET, significantly reduced the rate of cells in the $\mathrm{S}$ phase compared with the findings in the controls and in the samples treated with CRET alone (Fig. 3A, B and S2).

Based on these results, the expression of cyclin D1, a regulatory protein that participates in the progression of the cell cycle from the $G_{1}$ to $S$ phase, was analyzed. At $4 \mathrm{~h}$, treatment with sorafenib applied alone or in combination with CRET significantly reduced the expression of cyclin D1 compared with that of the controls, and the effect of the combined treatment was significantly stronger than that of CRET only. At the end of the 48-h treatment, only electrical treatment provoked a significant reduction in cyclin D1 expression (Fig. 3C and D).

Effects of sorafenib and CRET on ERK1/2 and EGFR. To study the potential early involvement of the MAPK/ERK $1 / 2$ signaling pathway in the effects observed on cell viability, the expression of the non-phosphorylated and phosphorylated forms of ERK1/2 and EGFR in response to short-term treatments, were analyzed by western blotting. After $4 \mathrm{~h}$ of exposure, electrical treatment decreased ERK1/2 phosphorylation (p-ERK1/2 over total ERK1/2 protein). This decrease was significantly higher in the combined treatment (Fig. 4A and C). On the other hand, the expression of the phosphorylated receptor (p-EGFR over total EGFR protein) was not significantly affected by the electrical treatment. In contrast, sorafenib and the combined treatment significantly reduced the expression of the phosphorylated receptor over controls (Fig. 4B and C)

\section{Discussion}

Electrothermal CRET therapies are currently used due to their reported analgesic, anti-inflammatory and tissue regenerative effects. The fact that these therapies are often recommended for patients with cancer who are also undergoing chemotherapy highlights the need to expand the currently insufficient knowledge about potential interactions between electrical and 
A

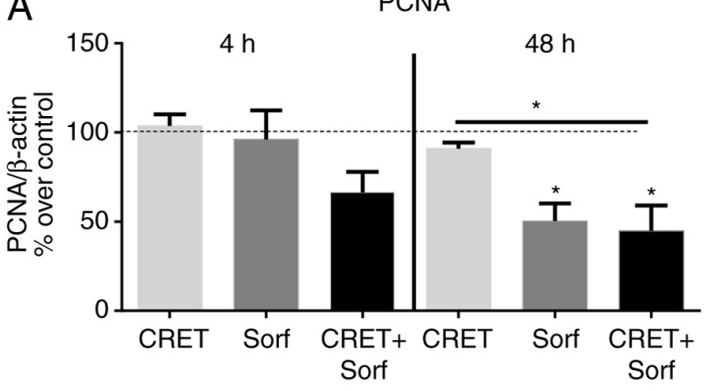

B

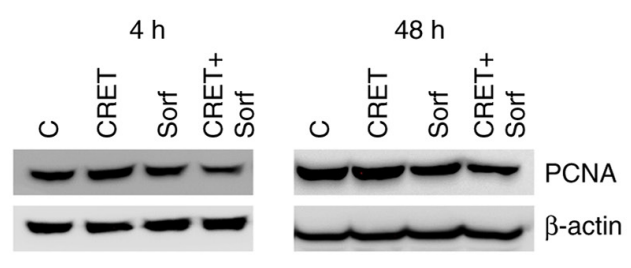

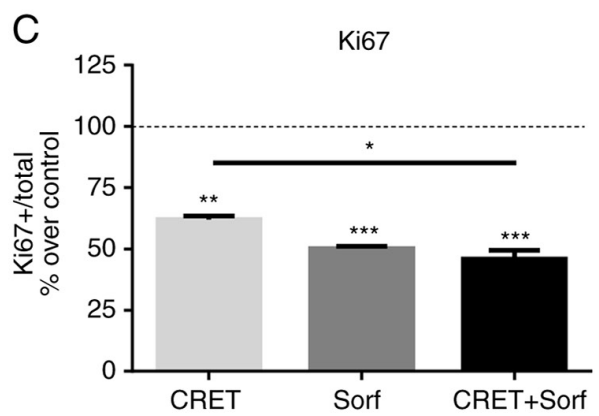
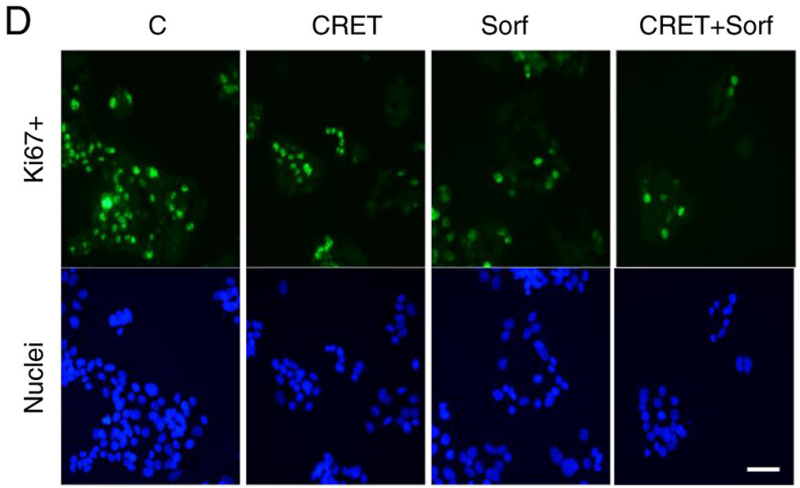

Figure 2. Effect of CRET and/or sorafenib treatment on the expression of the proliferation markers, PCNA and Ki67. (A) Western blotting of PCNA expression after treatment with CRET alone ( 4 or $24 \mathrm{~h}$ of intermittent exposure $+24 \mathrm{~h}$ post-exposure), sorafenib alone ( 4 or $48 \mathrm{~h}$ ) or in combination. Data are presented as the ratio of PCNA to $\beta$-actin (PCNA/ $\beta$-actin). All values represent the mean \pm SEM of $\geq 3$ experimental replicates. "P<0.05. (B) Representative western blots using $\beta$-actin as the loading control. (C) Immunofluorescence of Ki67 expression. Cells were treated with CRET alone ( $24 \mathrm{~h}+24 \mathrm{~h}$ post-exposure), sorafenib alone ( $48 \mathrm{~h}$ ) or in combination. ${ }^{*} \mathrm{P}<0.05,{ }^{* *} \mathrm{P}<0.01$ and ${ }^{* * *} \mathrm{P}<0.001$. Data were statistically analyzed using One-way ANOVA followed by Bonferroni post-hoc test. (D) Representative images of immunofluorescence for Ki67. Green represents Ki67-positive cells stained with anti-Ki67 antibody and Alexa Fluor $^{\circledast}$ 488. Blue represents nuclei stained with DAPI. Scale bar, $50 \mu \mathrm{m}$; same scale in all micrographs. CRET, capacitive-resistive electrothermal therapies; PCNA, proliferating cell nuclear antigen; Sorf, sorafenib; C, control.

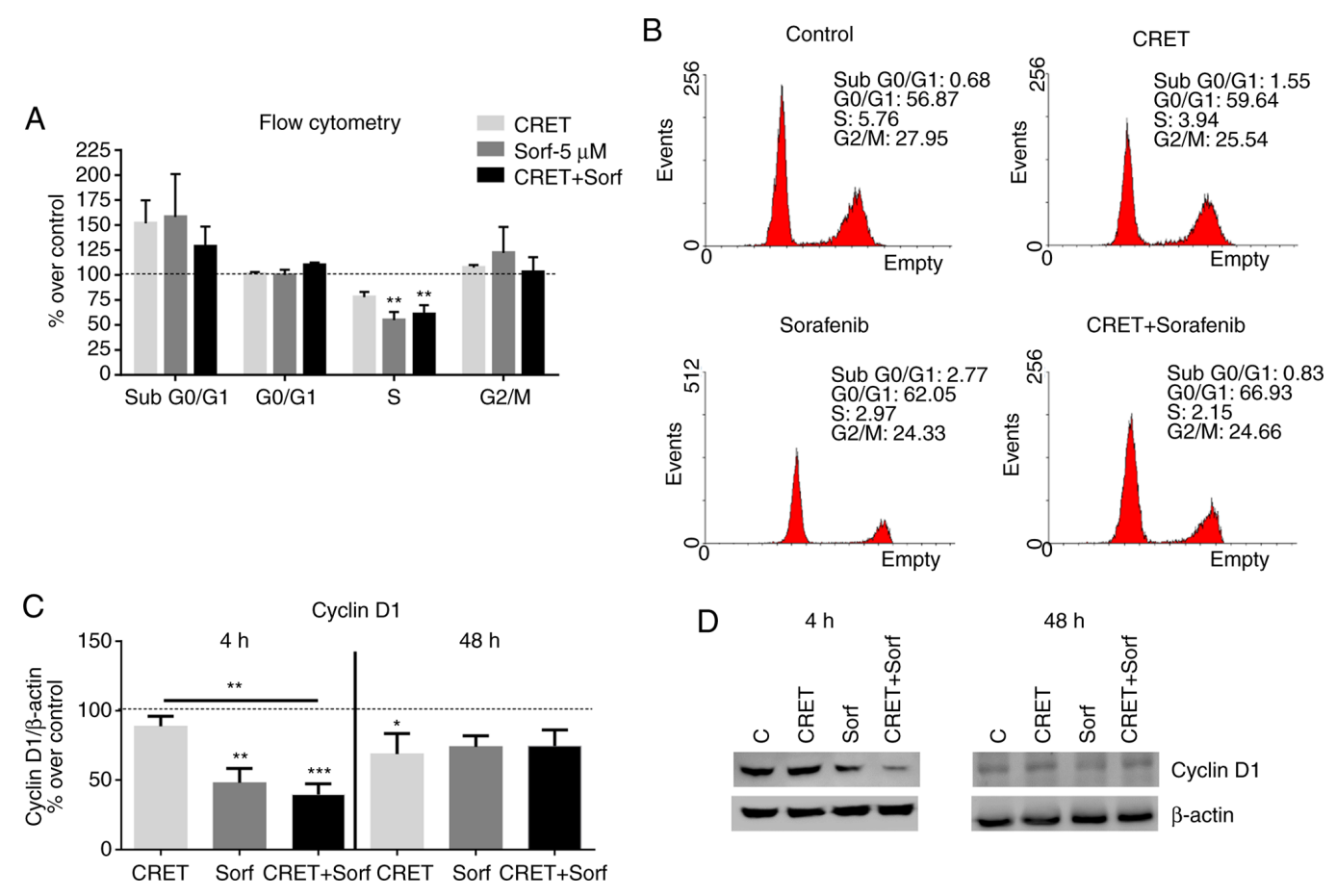

Figure 3. Effect of CRET and/or sorafenib on apoptosis and the cell cycle. (A) Flow cytometry analysis of samples treated with CRET alone (24 h intermittent exposure $+24 \mathrm{~h}$ post-exposure), sorafenib alone $(48 \mathrm{~h}$ ), or in combination. Data are presented as percentages of the mean \pm SEM of $\geq 3$ experimental replicates, and are normalized to the respective controls. ${ }^{* *} \mathrm{P}<0.01$. (B) Representative flow cytometry histograms of HepG2 cells treated with propidium iodide. (C) Western blotting of cyclin D1 expression after treatment with CRET alone ( 4 or $24 \mathrm{~h}$ intermittent exposure $+24 \mathrm{~h}$ post-exposure), sorafenib alone ( $4 \mathrm{or} 48 \mathrm{~h}$ ) or in combination. Data are presented as the ratio of cyclin D1 to beta-actin (cyclin D1/ $\beta$-actin). Data are presented as the mean \pm SEM of $\geq 3$ experimental replicates. ${ }^{*} \mathrm{P}<0.05,{ }^{* *} \mathrm{P}<0.01$ and ${ }^{* * * *} \mathrm{P}<0.001$. Data were statistically analyzed using One-way ANOVA followed by Bonferroni post-hoc test. (D) Representative western blots using $\beta$-actin as the loading control. CRET, capacitive-resistive electrothermal therapies; $\mathrm{C}$, control; Sorf, sorafenib. 
A

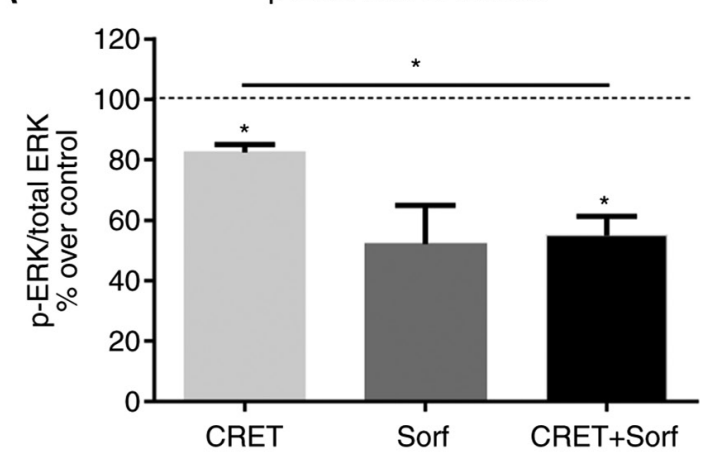

C

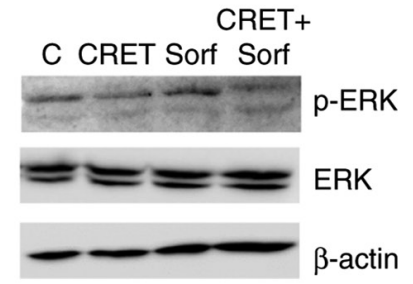

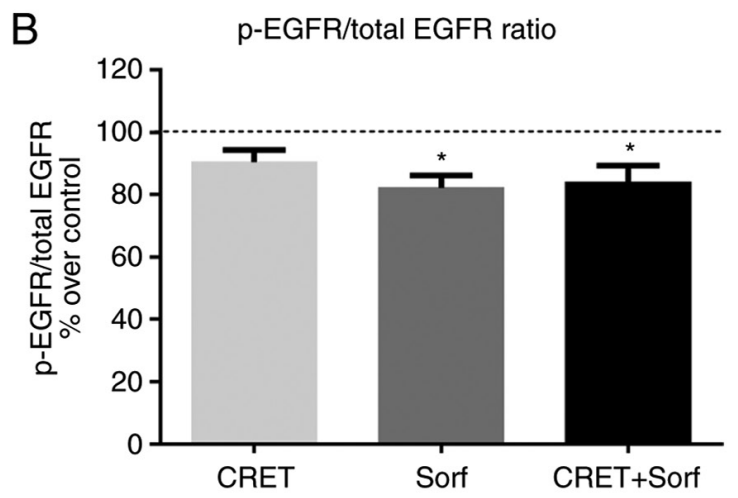

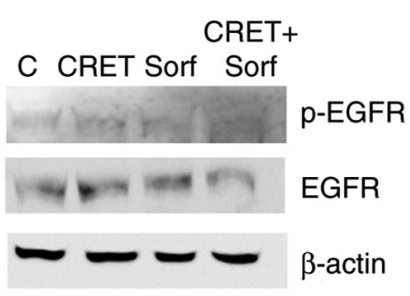

Figure 4. Effect of CRET and/or sorafenib on EGFR and ERK expression and activation. Western blotting results for p-ERK, ERK, p-EGFR and EGFR expression are presented. The samples were treated with CRET alone ( $4 \mathrm{~h}$ intermittent exposure), sorafenib alone (4 h) or in combination. Data are presented as the ratio of (A) p-ERK1/2 over total ERK1/2 protein and (B) p-EGFR over total EGFR protein. Data are presented as the mean \pm SEM of $\geq 3$ experimental replicates. * $\mathrm{P}<0.05$. Data were statistically analyzed using One-way ANOVA followed by Bonferroni post-hoc test. (C) Representative western blots using $\beta$-actin as the loading control. CRET, capacitive-resistive electrothermal therapies; p-, phosphorylated; Sorf, sorafenib; C, control.

chemical therapies. The present study investigated the effects of simultaneous treatment with the chemotherapeutic drug, sorafenib, and a $448-\mathrm{kHz}$ subthermal current on the proliferation and viability of the liver cancer cell line, HepG2. Our previous studies have shown that RF currents used in CRET therapies can induce, by themselves and under subthermal conditions, potential therapeutic responses, such as cell proliferation and differentiation in human adipose-derived stem cells (8-10).

Subthermal CRET treatments can also exert anti-proliferative and/or cytotoxic effects in neuroblastoma and HCC cell lines through altering the expression of proteins involved in the MAPK/ERK1/2 signaling pathway $(11,21)$. This pathway, when inhibited by sorafenib, restrains cell proliferation and induces apoptosis in HCC cells $(17,25)$. Thus, it is conceivable that, by exhibiting a mutual molecular target, synergistic, additive, enhancing, neutralizing or blocking interactions could occur between chemotherapeutic drugs and RF CRET currents. This possibility raises questions about the convenience of simultaneously exposing patients with cancer undergoing chemotherapy to CRET therapies for analgesic, anti-inflammatory or other purposes.

The present results support previously reported observations that, when applied separately, subthermal CRET $(1,21,26)$ and sorafenib $(27,28)$ can significantly reduce liver cancer cell viability. Besides, the present data showed that, at a concentration of $5 \mu \mathrm{M}$ sorafenib, the decrease in HepG2 viability after combined treatment with CRET + sorafenib was significantly larger than that induced when CRET or sorafenib were applied individually. This was suggestive of a cooperative effect of both treatments when combined, which could not be detected at lower or higher doses of the chemotherapeutic drug.
The analysis of apoptosis as a phenomenon potentially involved in the observed effects on the viability of HepG2 cells, did not reveal any significant differences in apoptosis rates, neither between the treated samples and their respective controls, nor between responses to different treatments. The lack of pro-apoptotic effects was consistent with previous results that reported that CRET induces cytostatic, but not cytotoxic, effects in HepG2 cells (26). Regarding sorafenib, the present results also reinforced previous observations about its lack of pro-apoptotic effects on HepG2 cells when administered at a concentration of $5 \mu \mathrm{M}$ (17).

The potential anti-proliferative effects of CRET and sorafenib, applied together or separately, were assessed by quantifying the expression of PCNA and Ki67, two specific proliferation-related antigens that are currently used as biomarkers for clinical prognosis in patients with $\operatorname{HCC}(29,30)$. PCNA is a nuclear antigen involved in DNA replication, molecular synthesis, mismatch repair and chromatin assembly (31). Ki67 is a nuclear protein that is only present in proliferating cells (32). During interphase, Ki67 is involved in the intracellular distribution of heterochromatin antigens and the nucleolar association of heterochromatin. During mitosis, Ki67 intervenes in the formation of the perichromosomal layer, thus preventing mitotic chromosome aggregation (33). The present results showed a significant reduction in the expression of both markers after $48 \mathrm{~h}$ of treatment with CRET only, with sorafenib only or with the combined treatment. Since preclinical tumor models demonstrated that silencing Ki67 or PCNA blocked cell cycle and proliferation (32), these results indicated that the three applied treatments may exert anti-proliferative effects on HepG2 cells. Furthermore, the decrease induced by the combined treatment in the expression 
of both proteins was significantly stronger than that elicited by CRET when administered alone. This may be indicative of a cooperative or synergistic action between the anti-proliferative effects of CRET and sorafenib when applied simultaneously.

The potential anti-proliferative effects were also investigated by analyzing the percentage of cells present in the different phases of the cell cycle. The data revealed a slight, non-significant increase in the fraction of cells in the $\mathrm{G}_{0} / \mathrm{G}_{1}$ phase after treatment with sorafenib only, which is consistent with previously reported results (34). In the absence of sorafenib, CRET induced a non-significant decrease in the percentage of cells in the $\mathrm{S}$ phase which is also consistent with previously reported data (1). By contrast, a significant decrease in the fraction of cells in the $\mathrm{S}$ phase was observed in samples subjected to combined treatment, which could be the result of an enhancing effect of weaker cytostatic responses potentially induced by each of both stimuli.

The cell cycle is regulated by a variety of proteins, including cyclin D1, which is involved in the progression of the cell cycle from the $\mathrm{G} 1$ to $\mathrm{S}$ phase. This cyclin forms active complexes with the cyclin-dependent kinases, CDK4 and CDK6, which promote cell cycle progression through phosphorylation and inactivation of the retinoblastoma protein (35). Since cyclin D1 is upregulated in hepatocarcinomas (36), its inhibition may be a useful chemo-preventative strategy for the treatment of this type of cancer. The decreased expression of cyclin D1 induced by the three treatments evaluated in the present study is consistent with the corresponding reductions observed in the rate of cells in S-phase. Taken together, these effects are consistent with the aforementioned anti-proliferative response. In line with these results, a previous study showed that CRET in combination with chemical drugs could induce anti-proliferative effects mediated by cell cycle arrest. Saitoh et al (15) reported that CRET and ascorbic acid synergistically inhibited the proliferation of Ehrlich ascites tumor cells through the generation of reactive oxygen species and inducing cell cycle arrest in the $\mathrm{G}_{2} / \mathrm{M}$ phase.

The possibility that the Ras/ERK1/2 signaling pathway may be involved in the anti-proliferative responses observed in the present study has been investigated. The Ras/Raf/MEK/ERK signaling cascade is an important MAPK signaling pathway. Various stimuli capable of activating cell surface receptors in turn activate this cascade, leading to the expression of a number of genes that regulate cell proliferation, differentiation and apoptosis. ERK regulates cell cycle progression from the G1 to $\mathrm{S}$ phase through activation of cyclin D1, and ERK phosphorylation has been shown to activate a variety of target molecules involved in liver cancer. Since the Raf kinase of the Ras/ERK signaling pathway is also known to be a molecular target of sorafenib (25), the present study investigated the involvement of the Ras/ERK signaling pathway in the effects of CRET, sorafenib and the aforementioned combined treatment, as well as the potential interactions between the chemical and electrical treatments via such pathway. For that purpose, the present study analyzed the expression levels of the active and non-active forms of ERK1/2 and EGFR, which is one of the receptors that activates the Ras/ERK1/2 signaling pathway (35). The anti-proliferative and angiogenic effects of sorafenib on HCC cells is mediated by the inhibition of the Raf, BRAF, VEGFR2, VEGFR3 and PDGFR pathways. Although the EGFR pathway would not be directly involved in these effects, it has been shown that the activation of this receptor modulates cellular sensitivity to sorafenib (36). The results obtained in the present study after $4 \mathrm{~h}$ of exposure to CRET, alone or in combination with sorafenib, revealed that the ratios $\mathrm{p}$-ERK1/2/total protein and $\mathrm{p}$-EGFR/total protein were downregulated, which indicated that these treatments induce a significant reduction in the activation, but not in the expression, of ERK1/2 and EGFR. These early effects, which could lead to a slower kinetics of the MAPK/ERK signaling pathway (25), would result in the anti-proliferative responses observed $44 \mathrm{~h}$ later. In addition, the decreased activation of EGFR induced by sorafenib or in combination, could promote a decrease in the resistance of HepG2 cells to sorafenib.

In conclusion, at the concentrations evaluated in the present study, no evidence was found to suggest that CRET exposure could compromise the anti-proliferative efficacy of sorafenib on HepG2 cells. Furthermore, at the standard pharmacological concentration of $5 \mu \mathrm{M}$, simultaneous treatment with CRET induced an anti-proliferative response in HepG2 cells that was significantly greater than that induced by each of the treatments when applied separately. Such increase could be due to a deceleration of the cell cycle, mediated at least in part by the decreased expression of cyclin D1, and a slowdown in the kinetics of the Ras/ERK1/2 signaling pathway. Taken together, these data do not support the hypothesis that CRET exposure may inhibit or diminish the effects of a chemotherapeutic drug used for cancer treatment.

Despite the demonstrated beneficial effects of sorafenib as a treatment for $\mathrm{HCC}$, a significant number of patients experience recurrence $(25,37,38)$. The present results and our previously reported data on the anti-proliferative response of human cancer cells to subtermal electrical stimulation, applied alone or in combination with chemical agents, are consistent with those from a number of experimental studies on therapeutic applications in oncology (39-41). Thus, innovative treatments, such as CRET plus chemotherapy, may offer novel possibilities for improving the survival of patients with cancer, and highlight the need for further research into novel therapeutic approaches for the treatment of cancer, such as those based on the combined action of electrical and chemical treatments.

\section{Acknowledgements}

Not applicable.

\section{Funding}

The present study was financially supported by the Foundation for Biomedical Research of the Ramón y Cajal University Hospital (FiBio-HRC Project; grant no. 2015/0050) and the European Defense Agency/Spanish Ministry of Defense (Project RF Biological Effects; grant no. MOU EUROPA ERG 101.013).

\section{Availability of data and materials}

The datasets used and/or analyzed in the current study are available from the corresponding author on reasonable request. 


\section{Authors' contributions}

MLHB, MAM, MAT and AU conceived and designed the experiments, analyzed and interpreted the data, and wrote the manuscript. MLHB, MAM, MAT, LM and ETM performed the experiments, and collected and analyzed data. MLHB, MAM and AU confirm the authenticity of all the raw data. All authors read and approved the final version of the manuscript.

\section{Ethics approval and consent to participate}

Not applicable.

\section{Patient consent for publication}

Not applicable.

\section{Competing interests}

The authors declare that they have no competing interests.

\section{References}

1. Hernández-Bule ML, Trillo MA, Cid MA, Leal J and Ubeda A: In vitro exposure to $0.57-\mathrm{MHz}$ electric currents exerts cytostatic effects in HepG2 human hepatocarcinoma cells. Int J Oncol 30: 583-592, 2007

2. Naranjo P, Lopez-Estebaranz J, Shoaib $\mathrm{T}$ and Pinto $\mathrm{H}$ : Non-ablative capacitive resistive $448 \mathrm{kHz}$ radiofrequency for wrinkle reduction pilot study. Aesthetic Med 6: 41-48, 2020.

3. Yokota Y, Tashiro Y and Suzuki Y: Effect of capacitive and resistive electric transfer on tissue temperature, muscle flexibility, and blood circulation. J Nov Physiother 7: 325-331, 2017.

4. Coccetta CA, Sale P, Ferrara PE, Specchia A, Maccauro G, Ferriero $\mathrm{G}$ and Ronconi G: Effects of capacitive and resistive electric transfer therapy in patients with knee osteoarthritis: A randomized controlled trial. Int J Rehabil Res 42: 106-111, 2019.

5. Pavone C, Romeo S, D'Amato F, Usala M, Letizia Mauro G and Caruana G: Does transfer capacitive resistive energy has a therapeutic effect on Peyronie's disease? Randomized, single-blind sham-controlled study on 96 patients: Fast Pain Relief. Urol Int 99: 77-83, 2017.

6. Bito T, Tashiro Y, Suzuki Y, Kajiwara Y, Zeidan H, Kawagoe M, Sonoda T, Nakayama Y, Yokota Y, Shimoura K, et al: Acute effects of capacitive and resistive electric transfer (CRet) on the Achilles tendon. Electromagn Biol Med 38: 48-54, 2019.

7. Fousekis K, Chrysanthopoulos G, Tsekoura M, Mandalidis D, Mylonas K, Angelopoulos P, Koumoundourou D, Billis V and Tsepis E: Posterior thigh thermal skin adaptations to radiofrequency treatment at $448 \mathrm{kHz}$ applied with or without Indiba ${ }^{\circledR}$ fascia treatment tools. J Phys Ther Sci 32: 292-296, 2020.

8. Hernández-Bule ML, Paíno CL, Trillo MÁ and Úbeda A: Electric stimulation at $448 \mathrm{kHz}$ promotes proliferation of human mesenchymal stem cells. Cell Physiol Biochem 34: 1741-1755, 2014.

9. Hernandez Bule ML, Angeles Trillo M, Martinez Garcia MA Abilahoud $\mathrm{C}$ and Ubeda A: Chondrogenic differentiation of adipose-derived stem cells by radiofrequency Electric stimulation. J Stem Cell Res Ther 7: 7, 2017

10. Hernández-Bule ML, Martínez-Botas J, Trillo MÁ, Paíno CL and Úbeda A: Antiadipogenic effects of subthermal electric stimulation at $448 \mathrm{kHz}$ on differentiating human mesenchymal stem cells. Mol Med Rep 13: 3895-3903, 2016.

11. Hernández-Bule ML, Medel E, Colastra C, Roldán R and Úbeda A: Response of neuroblastoma cells to RF currents as a function of the signal frequency. BMC Cancer 19: 889, 2019.

12. Trillo MÁ, Martínez MA and Úbeda A: Effects of the signal modulation on the response of human fibroblasts to in vitro stimulation with subthermal RF currents. Electromagn Biol Med 40: 201-209, 2021.

13. Hernández-Bule ML, Roldán E, Matilla J, Trillo MA and Ubeda A: Radiofrequency currents exert cytotoxic effects in NB69 human neuroblastoma cells but not in peripheral blood mononuclear cells. Int J Oncol 41: 1251-1259, 2012.
14. Kato S, Saitoh Y and Miwa N: Repressive effects of a capacitive-resistive electric transfer (CRet) hyperthermic apparatus combined with provitamin $\mathrm{C}$ on intracellular lipid-droplets formation in adipocytes. Int J Hyperthermia 29: 30-37, 2013.

15. Saitoh Y, Yoshimoto T, Kato S and Miwa N: Synergic carcinostatic effects of ascorbic acid and hyperthermia on Ehrlich ascites tumor cell. Exp Oncol 37: 94-99, 2015.

16. Méndez-Sánchez N, Vásquez-Fernández F, Zamora-Valdés D and Uribe M: Sorafenib, a systemic therapy for hepatocellular carcinoma. Ann Hepatol 7: 46-51, 2008.

17. Liu L, Cao Y, Chen C,Zhang X, McNabola A, Wilkie D, Wilhelm S, Lynch $M$ and Carter C: Sorafenib blocks the RAF/MEK/ERK pathway, inhibits tumor angiogenesis, and induces tumor cell apoptosis in hepatocellular carcinoma model PLC/PRF/5. Cancer Res 66: 11851-11858, 2006.

18. Ge S and Huang D: Systemic therapies for hepatocellular carcinoma. Drug Discov Ther 9: 352-362, 2015.

19. Llovet JM, Ricci S, Mazzaferro V, Hilgard P, Gane E, Blanc JF, de Oliveira AC, Santoro A, Raoul JL, Forner A, et al; SHARP Investigators Study Group: Sorafenib in advanced hepatocellular carcinoma. N Engl J Med 359: 378-390, 2008.

20. Wilhelm SM, Carter C, Tang L, Wilkie D, McNabola A, Rong H, Chen C, Zhang X, Vincent P, McHugh M, et al: BAY 43-9006 exhibits broad spectrum oral antitumor activity and targets the RAF/MEK/ERK pathway and receptor tyrosine kinases involved in tumor progression and angiogenesis. Cancer Res 64: 7099-7109, 2004.

21. Hernández-Bule ML, Trillo MÁ and Úbeda A: Molecular mechanisms underlying antiproliferative and differentiating responses of hepatocarcinoma cells to subthermal electric stimulation. PLoS One 9: e84636, 2014.

22. Omar HA, Tolba MF, Hung JH and Al-Tel TH: OSU-2S/Sorafenib synergistic antitumor combination against hepatocellular carcinoma: The Role of PKCס/p53. Front Pharmacol 7: 463, 2016.

23. Zhang SS, Ni YH, Zhao CR, Qiao Z, Yu HX, Wang LY, Sun JY, Du C, Zhang JH, Dong LY, et al: Capsaicin enhances the antitumor activity of sorafenib in hepatocellular carcinoma cells and mouse xenograft tumors through increased ERK signaling. Acta Pharmacol Sin 39: 438-448, 2018.

24. Nowak E, Kammerer S and Küpper JH: ATP-based cell viability assay is superior to trypan blue exclusion and XTT assay in measuring cytotoxicity of anticancer drugs Taxol and Imatinib, and proteasome inhibitor MG-132 on human hepatoma cell line HepG2. Clin Hemorheol Microcirc 69: 327-336, 2018.

25. Marisi G, Cucchetti A, Ulivi P, Canale M, Cabibbo G, Solaini L, Foschi FG, De Matteis S, Ercolani G, Valgiusti M, et al: Ten years of sorafenib in hepatocellular carcinoma: Are there any predictive and/or prognostic markers? World J Gastroenterol 24: 4152-4163, 2018.

26. Hernández-Bule ML, Cid MA, Trillo MA, Leal J and Ubeda A: Cytostatic response of $\mathrm{HepG} 2$ to $0.57 \mathrm{MHz}$ electric currents mediated by changes in cell cycle control proteins. Int J Oncol 37: 1399-1405, 2010.

27. Chai H, Luo AZ, Weerasinghe P and Brown RE: Sorafenib downregulates ERK/Akt and STAT3 survival pathways and induces apoptosis in a human neuroblastoma cell line. Int J Clin Exp Pathol 3: 408-415, 2010.

28. Yang Y, Qin S-K, Wu Q, Wang ZS, Zheng RS, Tong XH, Liu H, Tao $\mathrm{L}$ and $\mathrm{He} \mathrm{XD}$ : Connexin-dependent gap junction enhancement is involved in the synergistic effect of sorafenib and all-trans retinoic acid on HCC growth inhibition. Oncol Rep 31: 540-550, 2014.

29. Tiniakos DG and Brunt EM: Proliferating cell nuclear antigen and Ki-67 labeling in hepatocellular nodules: A comparative study. Liver 19: 58-68, 1999.

30. Ma S, Yang J, Li J and Song J: The clinical utility of the proliferating cell nuclear antigen expression in patients with hepatocellular carcinoma. Tumour Biol 37: 7405-7412, 2016.

31. Boehm EM, Gildenberg MS and Washington MT: The many roles of PCNA in eukaryotic DNA replication. Enzymes 39: 231-254, 2016.

32. Burkhart RA, Ronnekleiv-Kelly SM and Pawlik TM: Personalized therapy in hepatocellular carcinoma: Molecular markers of prognosis and therapeutic response. Surg Oncol 26: 138-145, 2017.

33. Sun $X$ and Kaufman PD: Ki-67: More than a proliferation marker. Chromosoma 127: 175-186, 2018.

34. Duval AP, Troquier L, de Souza Silva O, Demartines N and Dormond O: Diclofenac potentiates sorafenib-based treatments of hepatocellular carcinoma by enhancing oxidative stress. Cancers (Basel) 11: 11, 2019. 
35. Dhillon AS, Hagan S, Rath O and Kolch W: MAP kinase signalling pathways in cancer. Oncogene 26: 3279-3290, 2007.

36. Ji L, Lin Z, Wan Z, Xia S, Jiang S, Cen D, Cai L, Xu J and Cai X: miR-486-3p mediates hepatocellular carcinoma sorafenib resistance by targeting FGFR4 and EGFR. Cell Death Dis 11: 250,2020

37. Lei X-F, Ke Y, Bao T-H, Tang HR, Wu XS, Shi ZT, Lin J, Zhang ZX, Gu H and Wang L: Effect and safety of sorafenib in patients with intermediate hepatocellular carcinoma who received transarterial chemoembolization: A retrospective comparative study. World J Clin Cases 6: 74-83, 2018.

38. Falzone L, Salomone S and Libra M: Evolution of cancer pharmacological treatments at the turn of the third millennium. Front Pharmacol 9: 1300, 2018

39. Giladi M, Weinberg U, Schneiderman RS, Porat Y, Munster M, Voloshin T, Blatt R, Cahal S, Itzhaki A, Onn A, et al: Alternating electric fields (tumor-treating fields therapy) can improve chemotherapy treatment efficacy in non-small cell lung cancer both in vitro and in vivo. Semin Oncol 41 (Suppl 6): S35-S41, 2014.
40. Yang W-H, Xie J, Lai Z-Y, Yang MD, Zhang GH, Li Y, Mu JB and $\mathrm{Xu}$ J: Radiofrequency deep hyperthermia combined with chemotherapy in the treatment of advanced non-small cell lung cancer. Chin Med J (Engl) 132: 922-927, 2019.

41. Wust P, Stein U and Ghadjar P: Non-thermal membrane effects of electromagnetic fields and therapeutic applications in oncology. Int J Hyperthermia 38: 715-731, 2021.

c) (i) $\Theta$ This work is licensed under a Creative Commons Attribution-NonCommercial-NoDerivatives 4.0 International (CC BY-NC-ND 4.0) License. 